:urred with a frequency of $10^{-7}$ or ligher. Some of these colonies retained he ability to fix nitrogen when subsultured and in these cases maintenance of the $\mathbf{R}$ factor was not essential for etention of $\mathrm{N}_{2}$-fixing ability. Not unixpectedly, the $\mathrm{N}_{2}$-fixing Klebsiella uybrids acted like most naturallyxccurring free-living $\mathrm{N}_{2}$ fixers in fixing litrogen only under anaerobic confitions and in the absence of combined aitrogen.

The ability of Rhizobium strains to ix nitrogen only when within nodules on the roots of legumes has puzzled scientists for more than half a century. These bacteria grow in culture media sut have never been shown to fix aitrogen in vitro, a fact which has in the past often prompted the suggestion that the legume plant contributes at least part of the genes concerned with fixation. Dunican and Tierney suggest that the $\mathrm{N}_{2}$-fixing ability is present though not phenotypically expressed in $R$. trifolii and this ability is expressed when transferred to a new bacterial host. The alternative explanation that genetic material transferred from the Rhizobium activates certain latent $\mathrm{N}_{2}$-fixing genes in $K$. aerogenes cannot be entirely ruled out.

It is anticipated that this genetic system may be useful in studies of the physiology of legume root nodules and may also provide a means of improving strains of Rhizobium used in agriculture as seed inoculants. Legumes such as soybeans fix only about one quarter of their nitrogen requirements and a significant improvement in field fixation rates may be possible.

\section{Radius of neutron distribution in lead}

\section{from Peter E. Hodgson \\ Nuclear Theory Correspondent}

DURING the past few years there has been great interest in determining the sizes of atomic nuclei, and in particular the relative spatial extents of the constituent neutron and proton distributions. The proton distributions can be determined accurately from analyses of electron elastic scattering and muonic $X$ rays, but the neutron distributions can only be determined indirectly, and the results are less accurate due to the lack of knowledge of the nucleon-nucleon interaction. Many different methods have been used, and the results are in substantial agreement.

On the whole it is found that the proton and neutron distributions are quite similar, with perhaps a slight tendency for the neutron distribution to extend further from the centre of the nucleus than the proton distribution, particularly for heavier nuclei. There is also the possibility of a 'neutron halo', a very diffuse neutron cloud extending far beyond the nuclear surface, where protons cannot reach due to the Coulomb field.

A quantity of particular interest is the ratio of the root mean square neutron and proton radii, as this is a convenient way of specifying the relative extents of the neutron and proton distributions. Recent work has tended to give a value of about 1.05 for this ratio though some studies have given values below unity.

A recent measurement of this quantity has used the $(p, n)$ reaction to the isobaric analogue state of the target nucleus. This reaction concerns principally the outer neutrons and protons, and so is particularly sensitive to their densities in the surface of the nucleus. The final state has the same structure as the initial state and differs from it only by the substitution of a proton for a neutron in the highest quantum state. Another way of describing this is to say that the final state is formed from the initial state just by an isospin flip that changes a neutron into a proton. The interaction is thus brought about only by the isospin component of the nucleon-nucleon interaction, which determines the isospin term in the nucleon optical potential. Also affecting the isospin potential is the difference between the neutron and proton density distributions, so the reaction is sensitive to just the physical quantities of interest. All the interactions responsible for the $(\mathrm{p}, \mathrm{n})$ reaction are quite well known, and so is the proton distribution, so comparison between the experimental data and a series of neutron density distributions enables the latter to be determined.

The dependence of the cross section of these quantities is particularly simple if the distorted wave Born approximation is used. Then the cross section is proportional to the square of the transition amplitude

$$
\begin{gathered}
T(\mathrm{p}, \mathrm{n})=\left[2(N-Z)^{\frac{1}{2}} / A\right] \\
\int \chi_{\mathbf{n}}-(r) U_{\mathrm{i}}(r) \chi_{\mathrm{p}}{ }^{+}(r) d \mathbf{r}
\end{gathered}
$$

where $\chi_{\mathrm{n}}-(r)$ and $\chi_{\mathrm{p}}{ }^{+}(r)$ are the waves describing the outgoing neutron and incoming proton respectively and the isospin potential

$$
\begin{array}{r}
U_{\mathrm{i}}(r)=[A /(N-Z)] \int u_{\mathrm{t}}\left(\left|\mathbf{r}-\mathbf{r}^{\prime}\right|\right) \\
\left\{\rho_{\mathrm{n}}\left(r^{\prime}\right)-\rho_{\mathrm{p}}\left(r^{\prime}\right)\right\} d \mathbf{r}^{\prime}
\end{array}
$$

where $u_{\mathrm{t}}\left(\left|\mathbf{r}-\mathbf{r}^{\prime}\right|\right)$ is the isospin component of the nucleon-nucleon interaction and $\rho_{\mathrm{n}}\left(r^{\prime}\right)$ and $\rho_{\mathrm{p}}\left(r^{\prime}\right)$ are the neutron and proton density distributions respectively.

Schery, Lind and Zafiratos (Phys. Rev., C9, 416; 1974) have measured the cross section for the $(p, n)$ reaction to the isobaric analogue state of ${ }^{208} \mathrm{~Pb}$ at $25.8 \mathrm{MeV}$. They calculated the cross section from the above formulae using standard values of $u_{t}\left(\left|\mathbf{r}-\mathbf{r}^{\prime}\right|\right)$ and the optical potentials that fix the distorted waves $\chi_{\mathrm{n}}-(r)$ and $\chi_{\mathrm{p}}{ }^{+}(r)$. They assumed that the neutron density distribution has the form

$$
\rho_{\mathrm{n}}(r)=[1+\exp ((r-R) / a)]^{-1}
$$

and varied $R$ and $a$ to optimise the fit to the measured $(p, n)$ cross section. The best fit accounted well for the angular variation of the crosssection, which confirms the reliability of the model. The optimum values of the parameters $R$ and $a$ correspond to a ratio of the root mean square radii $\left\langle r_{\mathrm{n}}{ }^{2}\right\rangle^{1 / 2} /\left\langle r_{\mathrm{p}}{ }^{2}\right\rangle^{1 / 2}=$ $1.07 \pm 0.03$, thus confirming previous work indicating a slightly larger neutron distribution. Since $\left\langle r_{\mathrm{p}}{ }^{2}\right\rangle^{1 / 2}$ is $5.30 \mathrm{fm}$, the value of $\left\langle r_{\mathrm{n}}{ }^{2}\right\rangle^{1 / 2}$ is $5.68 \mathrm{fm}$.

The stability of this result with respect to small changes in the assumed potentials was investigated, and this accounts for part of the suggested $3 \%$ uncertainty. Studies of the effect of the difference between the unbound proton wave function in the final state of ${ }^{208} \mathrm{Bi}$ compared with the corresponding bound neutron wave function in ${ }^{208} \mathrm{~Pb}$ showed that the longer tail of the former reduces the ratio of root mean square radii by about $1 \%$. The effects of two step contributions to the reaction was also studied and found to be small.

\section{Plant messenger RNA and poly(A)}

from our

Plant Cell Physiology Correspondent

THE discovery that many animal messenger RNA molecules contain regions of poly(adenylic) acid is of considerable interest (see Nature, 247,426 and $512 ; 1974$ ). One reason for this is that the presence of $\operatorname{poly}(\mathrm{A})$ confers specific properties on mRNA molecules which can be exploited during RNA purification. Many workers have used columns of oligo(dT) cellulose or poly $(U)$ sepharose which retain the mRNA by specifically base pairing with the poly(A) region. Another approach has been to use unmodified cellulose or nitrocellulose to purify the mRNA.

A recent report shows that presumptive mRNA from plants, long known for its high content of adenylic acid, contains poly(A) tracts (Higgins et al., Nature new Biol., 246, 68; 1973). Sagher et al. (Biochim. biophys. Acta, 349, 32; 1974) now suggest that the length of poly(A) in higher plants is of the order of 150--250 nucleotides which contrasts with the 50 or so residues found attached to yeast and fungal mRNA (McLaughlin et al., J. biol. Chem., 248, 1466; 1973; Reed and Wintersberger, FEBS Lett., 32, 213; 1973; Silver and Horgen, Nature, 249, 252; 1974). At present only the poly(A)-containing RNA from polyribosomes of plants which synthesise a large number of proteins has been analysed and not surprisingly it is heterodisperse. Individual mRNAs of known function have yet to be isolated but exploitation of the poly(A) tag may speed up the process. Although this approach to the study of mRNA metabolism holds out great promise it should not be accepted 